\title{
Vers des réponses ciblées aux problèmes des situations d'urgence pour les maladies rares : présentation de la situation actuelle en France
}

Christophe Duguet

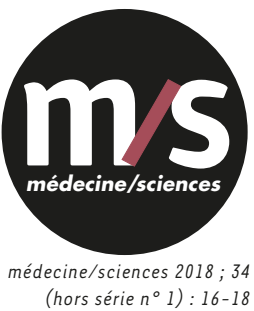

> Dans le cadre de la préparation du $3^{e}$ Plan Maladies rares, un petit groupe de travail multi-acteurs s'est penché sur la question spécifique des urgences maladies rares afin de faire un bilan et des propositions. Ce sont ces travaux qui sont présentés.

Chaque année, les services d'urgence gèrent 20 millions de passages. Aucun codage ni aucune statistique ne permet de connaître la part des personnes atteintes de maladies rares dans ces passages. Au regard de la prévalence de ces dernières et de la situation particulièrement grave d'un certain nombre de malades, on peut estimer à un million le nombre de passages de personnes concernées par des maladies rares chaque année. Certaines personnes sont concernées par une maladie rare et passent aux urgences pour une question liée à cette maladie. Pour d'autres, il peut s'agir de problèmes liés à d'autres événements de la vie, d'autres accidents, voire d'autres maladies. Quelle que soit la raison de cette urgence, certains actes fréquents ou courants en service d'urgence peuvent s'avérer néfastes pour certaines maladies rares. II faut toutefois se garder des généralisations. Toutes les personnes concernées par des maladies rares ne nécessitent pas des spécificités de prise en charge en urgence. Par ailleurs, beaucoup sont concernés par des problèmes inhérents aux urgences, comme les phénomènes de saturation, mais toutes n’ont pas des problèmes liés aux spécificités de leur maladie rare.

\section{Les difficultés rencontrées par les malades en situation d'urgence}

Les associations de malades concernés par des maladies rares sont très nombreuses à témoigner de cas précis et de difficultés fréquentes rencontrées aux urgences. Les médecins de centres de référence dans de nombreuses filières constatent eux aussi les conséquences de certaines prises en charge inadaptées. Les urgentistes ont souvent du mal à avoir rapidement accès aux informations pertinentes pouvant leur permettre d'adapter leurs pratiques à des maladies qu'ils n'ont jamais rencontrées et qu'ils ne rencontreront parfois qu'une seule fois dans l'ensemble de leur vie professionnelle. Cette méconnaissance des professionnels peut générer des pratiques inadaptées, pouvant elles-mêmes déboucher sur des accidents médicaux graves. Dans un certain nombre de cas, ces derniers auraient pu être évités si la communication avec le malade

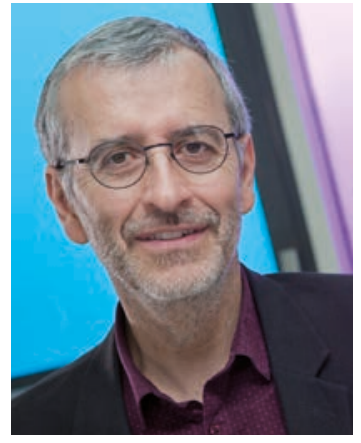

Directeur des Affaires Publiques, AFM-Téléthon, 1 , rue de l'Internationale, 94100 Évry, France. cduguet@afm-telethon.fr

et sa famille avait mieux fonctionné. En situation d'urgence, la communication entre malade et professionnel de santé n'est pas toujours facile. II n'est pas aidé de faire entendre sa spécificité et son expertise de la maladie quand on est malade ou famille en situation d'urgence. Le problème n'est pas nouveau, mais malgré l'amélioration indéniable de l'organisation des soins pour les maladies rares, il ne semble pas diminuer. Il semblerait même que les difficultés aient tendance à se multiplier. Cela peut être dû aux difficultés générales d'engorgement de certains services d'urgence, mais aussi à d'autres raisons, notamment une contrepartie de l'amélioration de la prise en charge de nombreuses personnes malades concernées par des maladies rares: elles vivent plus longtemps, y compris à leur domicile, dans des situations médicales très complexes, ce qui génère des situations de décompensation qui peuvent être encore plus difficiles à gérer en situation d'urgence. De plus en plus de personnes concernées par les maladies rares voyagent loin de leur réseau habituel de prise en charge médicale.

Les difficultés sont fréquentes, réelles, responsables de pertes de chance parfois importantes pour un très grand nombre de malades et génèrent certainement des dépenses de santé inutiles. Dès le premier Plan Maladies rares, alors que la question de l'organisation et de la lisibilité du système de soins hospitalier n'était pas aussi avancée qu'à l'heure actuelle, la question 
des urgences avait déjà été identifiée comme une priorité. Dans le contexte de l'époque, la stratégie avait presque exclusivement été celle de l'amélioration de l'information à apporter aux urgentistes, par le biais des patients eux-mêmes, par le biais des cartes malades et par l'information des professionnels sur les gestes à faire ou à ne pas faire, avec la création d'Orphanet urgence.

\section{La solution des cartes de soins et d'urgence}

En tout, 22 cartes officielles de soins et d'urgences maladies rares ont été créées par la Direction Générale de la Santé (DGS), mais n’ont jamais été mises à jour et les stocks sont, depuis longtemps, épuisés. Elles continuent malgré tout d'être plébiscitées par les malades qui ont eu la chance d'en bénéficier. Une évaluation externe de ces cartes a été financée à la fin du premier plan maladies rares par le ministère de la Santé. Cette évaluation a conclu à l'utilité de cette carte, sous réserve de les simplifier pour éviter la confusion entre soins courants et information sur les situations d'urgence. Sur cette base, le Plan National Maladies Rares 2 (PNMR 2) a prévu une multiplication et une généralisation de cartes à tous les malades ayant besoin de prises en charge spécifiques. Cette mesure s'est malheureusement enlisée pendant le second PNMR et aucune nouvelle carte n'a été créée.

La Direction Générale de l'Organisation des Soins (DGOS) a décidé il y a quelques semaines de relancer le processus en proposant à toutes les filières maladies rares l'impression d'un nouveau modèle-type simplifié au format carte de crédit, avec deux pages générales et deux pages centrales à personnaliser en fonction de chaque filière. Espérons que cette nouvelle stratégie sera plus efficace. Elle ne pourra l'être que si les associations de malades sont très étroitement impliquées dans la production de ces cartes, et notamment les pages centrales. N'oublions pas que les malades les portent et sont confrontés aux difficultés de les faire prendre en compte. Ces nouvelles cartes, très simplifiées, devront être évaluées pour voir si elles répondent réellement à leur objet.

\section{L'apport d'Orphanet Urgences}

Orphanet Urgences est une collection de recommandations pratiques pour la prise en charge des malades atteints d'une maladie rare et qui nécessitent des soins médicaux spécifiques en urgence. Ces documents sont accessibles en ligne sur un même site. Ces outils sont destinés, d'une part aux médecins urgentistes qui interviennent sur le lieu d'urgence, notamment la régulation SAMU, et d'autre part aux urgences hospitalières. Les recommandations sont élaborées avec les centres de référence maladies rares, la Société française de médecine d'urgence, l'Agence de biomédecine et les associations de malades concernées. Elles sont aujourd'hui traduites et adaptées en sept langues. Dix ans après leur lancement, elles sont au nombre de 80 . Ces outils sont très utiles, pertinents et performants. II n'existe pas d'étude suffisamment précise pour identifier le nombre de fiches à réaliser pour couvrir l'ensemble des maladies rares nécessitant des prises en charge spécifiques en urgence, mais le besoin est nettement supérieur aux 80 existantes.
Les moyens aujourd'hui disponibles pour financer cette activité ne permettront pas d'espérer que l'ensemble des maladies qui en auraient besoin soit couvert par des fiches Orphanet Urgences.

Des cartes malades papier, des fiches Orphanet Urgences et quelques actions de sensibilisation des urgentistes forment donc le bilan de la politique volontariste impulsée par les deux premiers plans maladies rares. Il convient d'y ajouter les initiatives isolées, mais très intéressantes prises par certaines filières ou centres de référence, comme la mise en place d'hotline pour répondre aux situations d'urgences ou d'initiatives, tout aussi intéressantes, prises par des associations de malades.

\section{Le dispositif « patients remarquables 》}

En fonction des territoires et des dynamiques locales, des patients maladies rares ont pu être intégrés dans des dispositifs dans des dispositifs locaux dits de «patients remarquables », c'est-à-dire de patients dont la situation médicale comporte des spécificités à prendre en compte absolument lors d'un passage aux urgences. Etre répertorié comme tel permet d'être identifié en amont, avant d'avoir eu un problème d'urgence, par les services d'urgence. Ainsi, lorsque l'urgence se produit, les meilleures informations peuvent être données à l'urgentiste qui assure la prise en charge. Différentes expériences existent également, qui accompagnent cette identification dans ces fichiers «patients remarquables » du dossier de liaison d'urgence (DLU), pour orienter au mieux et rapidement le malade concerné par une situation d'urgence. Dans un certain nombre de cas, la meilleure prise en charge en urgence consiste justement de ne pas passer par le service des urgences, mais à aller directement vers le service spécialisé capable d'assurer la meilleure prise en charge.

Malheureusement, ce dispositif de patients remarquables est plus ou moins opérationnel suivant les départements et reste par nature départemental. Enfin, ce dispositif est peu connu des acteurs maladies rares et peu sollicité.

Même si les moyens suffisants étaient affectés à sa mise en œuvre, cette stratégie reste insuffisante pour espérer qu'à court terme aucun patient ne connaisse plus en situation d'urgence une perte de chance de la rareté de sa maladie. Au-delà de l'amélioration nécessaire des outils d'information et de la production et mise à disposition des bonnes pratiques, il faut aujourd'hui recourir à de nouveaux leviers. Le premier est le principe qu'une urgence non programmée se prépare et s'anti- 
1 - Réaliser un état des lieux des besoins

- Action 1 : Enquêtes

2 - Améliorer et multiplier les outils d'information et de sensibilisation

- Action 2 : Produire, diffuser et systématiser des cartes d'Urgence

- Action 3 : Couvrir en «fiches Orphanet Urgences», toutes les maladies pour lesquelles il y a un besoin

- Action 4 : PNDS et Urgence

- Action 5 : Création d'une base de données médicaments, maladies rares en situation d'urgence

- Action 6 : Création d'un kit Urgence pour les patients

- Action 7 : Soutenir les actions d'information et de sensibilisation des associations de malades

3 - Développer et généraliser le dispositif patient remarquable et le Dossier de Liaison d’Urgence

- Action 8 : Intégrer au plus vite la prise en compte des spécificités maladies rares dans le développement du SI SAMU

4 - Soutenir la création de Hotlines - Urgences Maladies Rares

- Action 9 : Clarifier les conditions financières de mise en œuvre des Hotlines maladies rares et les soutenir au titre d'actions prioritaires des plans d'action annuels des FSMR

5 - Mettre en œuvre des plans d'action Urgence MR dans chaque région

- Action 10 : Mise en œuvre de plans d'action d'urgence maladies rares dans chaque région

Figure 1. Proposition d'un plan d'action «Urgence et maladies rares ».

cipe. Il s'agit ensuite d'introduire une nouvelle logique: la meilleure prise en charge en situation d'urgence nécessite que l'urgentiste n'ait pas simplement une connaissance des spécificités de la maladie, mais aussi des spécificités de la personne malade concernée par la maladie rare, c'est-à-dire avoir les informations les plus personnalisées possible. Le troisième levier serait de se saisir des opportunités de réforme en cours des systèmes d'information médicale, tout particulièrement celui des SAMU. Enfin, il faut prendre conscience qu'un tel enjeu se pilote au niveau national comme régional. Sans identification de pilote devant assurer cette mission, la situation a peu de chance d'évoluer.

\section{Un nécessaire plan d'action}

Sur cette base, le groupe de travail précédemment mentionné a proposé pour le PNMR 3 un plan d'action articulé autour de cinq axes et dix actions (Figure 1). Parmi ces mesures, il s'agit de poursuivre ce qui a été fait, en accordant les moyens nécessaires. D'autres consistent à rendre obligatoire dans chaque Protocole National de Diagnostic et de Soin (PNDS) un passage consacré aux urgences, voire directement la production des fiches Orphanet Urgences dans chaque PNDS, la création d'un kit Urgence pour les patients à diffuser, afin de les aider à anticiper les situations d'urgence, le soutien des actions d'information et de sensibilisation des associations de malades, l'intégration au plus vite de la prise en compte des spécifications maladies rares dans le développement du Système d'Information SAMU, la clarification des conditions financières de mise en œuvre des hotlines maladies rares et leur soutien, enfin la mise en œuvre des plans d'action d'urgence maladies rares dans chaque région.

En conclusion, améliorer réellement la qualité et l'efficience des parcours aux urgences des personnes concernées est possible, peu coûteux et très efficace, tant en limitation de perte de chance pour les malades que d'économies pour le système hospitalier. II manque une vraie prise de conscience qu'il est de l'intérêt de tous de faire le choix politique d'un plan d'action global, cohérent et réellement piloté. $\diamond$

Towards targeted answers to emergency issues

for rare diseases

\section{LIENS D'INTÉRÊT}

L'auteur déclare n'avoir aucun lien d'intérêt concernant les données publiées dans cet article. 